\title{
Communication Delays in Wide Area Measurement Systems
}

\author{
Biju Naduvathuparambil, Matthew C. Valenti, and Ali Feliachi \\ Lane Dept. of Comp. Sci. \& Elect. Eng. \\ West Virginia University \\ Morgantown, WV 26506-6109
}

Key Words: Phasor Measurement Units, Wide Area Measurement Systems, Communication Delay, Power System Stability, State Estimation, Adaptive Relaying

\begin{abstract}
This paper provides a tutorial introduction to phasor measurement units (PMU) when applied in a power system environment, provides an overview of communication alternatives for wide area measurement systems (WAMS), and computes the delay budget for each type of communication link. The goal of this study is to provide data regarding the communication delay that can be incorporated into the analysis and simulation of WAMS.
\end{abstract}

\section{INTRODUCTION}

The growing trend towards restructuring the power industry and the ever increasing demand for power exchange calls for employing wide area measurement systems (WAMS) for almost real-time measurements that could be used to maintain or improve the stability of the system. Conventional SCADA (supervisory control and data acquisition) / EMS (energy management systems) stability control systems do not provide efficient solutions in the case of cascaded outages as these systems are designed to act locally, based on set operational parameters [1]. These systems do not provide for dynamic coverage of a wide area power network. WAMS, on the other hand, provide for a dynamic coverage of the network and are able to handle cascaded outages through coordinated and optimized stabilizing actions. Emerging techniques like phasor measurement units (PMUs) are being used in WAMS, and these complement conventional SCADA / EMS systems thereby providing a wide area system view and increased system stability.

The general concept of a wide area measurement system is introduced in Section II. It also provides an insight into the operating technique of a PMU followed by the applications of a PMU in a WAMS environment. This section also introduces the IEEE 1344 data format used by the PMU. Communication issues and related communication link delay budgets for various types of wired and wireless communication links are presented in Section III. Finally, Section IV concludes the paper.

\section{Concept of Wide Area Measurement SYSTEMS}

\section{A. General System Setup}

In WAMS, PMUs are strategically placed throughout a wide coverage area. The PMUs form part of local devices called system protection terminals (SPT)[1]. SPTs are able to run complete or parts of distributed control algorithms and can communicate directly with other SPTs, substation equipment and system protection centers (SPC), which are responsible for monitoring and control of the power grid. Communication delay plays a crucial role in WAMS by determining the time-lag before control action is initiated to dampen power system oscillations.

Fig. 1 represents the concept of WAMS. The PMUs measure voltage, current, and frequency phasors using the discrete Fourier transform (DFT) and can detect transients or surges within milliseconds of their occurrence [2]. PMUs use the IEEE 1344 data format for communication with the central monitoring station. In addition to the propagation delay of the particular link, the message format of the PMU and data rate of the link determine the communication delay in the system. Furthermore, there is also a processing delay due primarily to the window size of the DFT.

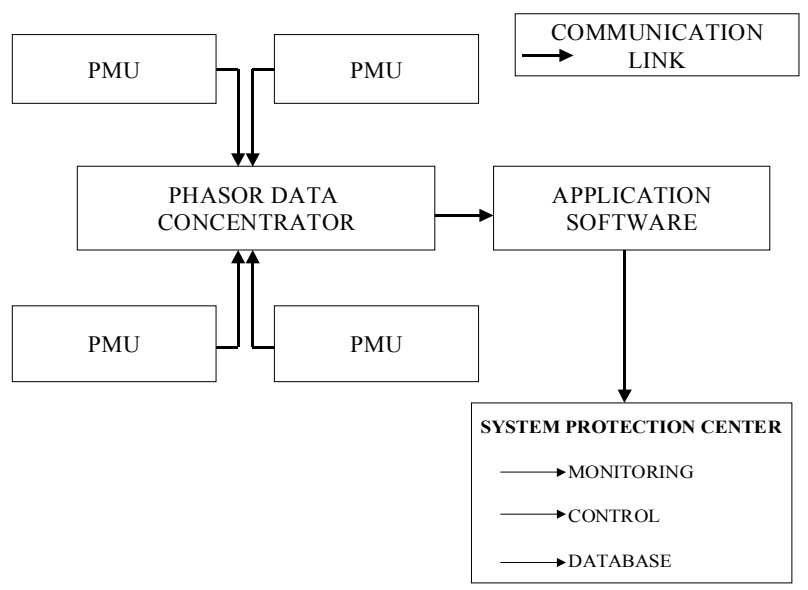

Fig. 1. Placement of PMUs in WAMS 


\section{B. Operating Principles of a PMU}

As indicated by Fig. 2, the analog power signal is converted into digital data by the analog to digital converter. For example, if we want to measure the voltage of the signal, then samples are taken for each cycle of the waveform and then the fundamental frequency component is calculated as follows (DFT)[2]:

$$
X=\frac{\sqrt{2}}{N} \sum_{k=1}^{N} x_{k} e^{-j k\left(\frac{2 \pi}{N}\right)}
$$

where $N$ is the total number of samples in one period, $X$ is the phasor and $x_{k}$ are the waveform samples.

From the above expression, we can see that the phasor information is dependent on the number of samples of the waveform and will give a correct representation of the fundamental frequency component even in the presence of transient signals. The samples are taken over one cycle or over multiple cycles of the power signal. Data windowing is used to smoothen the frequency spectrum and prevent / reduce overshoot of the signal caused due to discontinuities of the signal in the time domain (Gibbs phenomenon).

Using the DFT to calculate phasors is a better technique than using the waveform's zero-crossing. The problem with the zero-crossing technique is that the zerocrossings could be distorted (and could vary) due to the presence of harmonics and noise, thus affecting the reliability of the measuring unit. The positive sequence phasor can be calculated as follows [2]:

$$
X_{1}=\frac{1}{3}\left(X_{a}+\alpha X_{b}+\alpha^{2} X_{c}\right)
$$

where $\alpha=e^{\frac{j 2 \pi}{3}}$ and $X_{a}, X_{b}$, and $X_{c}$ are the DFT phasor coefficients of each of the three phases.

The PMU derives its inputs from the secondary sides of the three phases of a potential or current transformer and outputs the corresponding positive sequence voltage or current phasor. In the context of power system monitoring and control, positive sequence measurements are used more often than the three phase quantities. The complex valued phasors calculated do have a small uncertainity regarding their precise magnitude and angle. This is due primarily to the presence of noise in the analog input signal. The source of this noise could be the channel or the electronic equipment related to the power network. The sampling rate can be increased, in the presence of a large region of uncertainty, in order to minimize measurement errors. Longer data windows are also used to reduce errors. It has been found out that the error in estimation is inversely proportional to the square-root of the length of the data window.

\section{Applications of PMUs in WAMS Environment}

WAMS provide for improved stability and reliability based on the operational features of the PMU. Main applications of PMUs include:

State estimation: State estimation is the most important part of monitoring a power system [3]. PMUs can relay information on a continuous basis to the data acquisition centers, which can then generate a state vector that will follow the dynamics of the system [4]. With normal dedicated communication circuits operating at 4800 or 9600 baud, a continuous data stream of one phasor measurement every $2-5$ cycles $(33.3-83.33 \mathrm{msec})$ can be sustained [2].

Instability prediction: Presently, stability analysis of power systems is being done off-line. Synchronized phasor measurements can enable real-time stability analysis and instability prediction.

Adaptive relaying: Out-of-step relays detect power swings on the grid with the help of apparent impedance changes observed by a distance relay [5]. Presently, these out-of-step relays are set based on data observed from stability simulations that are conducted for all reasonable contingencies. The problem with this technique is that the actual conditions may vary as compared to the simulations that were performed for stability testing due to power swings. PMUs can be used to dynamically set the relays thus enabling a better response to power swings [6]. Improved control of power systems: Controllers like variable series capacitors (VSC), universal power flow controllers (UPFCs) and power system stabilizers regulate the grid based on a local feedback, which is then processed based on set operational parameters to ensure that the system working meets the objectives laid out. The problem here is that these models may not represent the actual conditions that could exist. Synchronized pha-

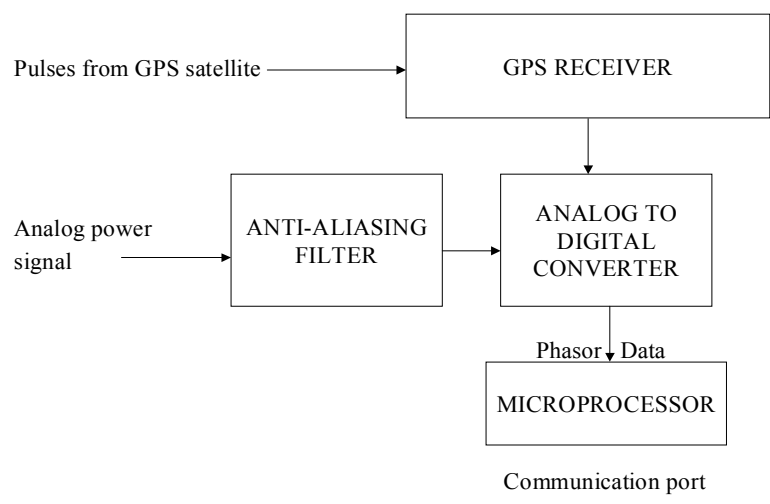

Fig. 2. Basic block diagram of a PMU 
sor measurements could provide direct feedback to these controllers, thereby providing for dynamic control of the power system.

\section{The IEEE Data Format}

The IEEE 1344 standard adresses issues like synchronization of data sampling, data to phasor conversions, and formats for timing input and phasor data output from the PMU [7].The standard not only helps in establishing a basis for phasor measurements, but also facilitates exchange of data between users who are involved in monitoring and control.

The PMU transmits messages (according to the IEEE 1344 format) in three types of frames:

Data frame: The data frame provides information regarding phasor data and the state of the digital inputs on each channel. It also defines the trigger status of frequency, angle, over-current, under-voltage and rate of frequency change.

Header frame: The header frame is an ASCII file that contains identification information about the PMU, data source, algorithms, and the analog filters used.

Configuration frame: This is a machine-readable binary file that contains PMU identification code, number of phasors, number of digital channels, channel and phasor names, nominal line frequency, and the transmission period of the phasors.

The PMU also receives messages that indicate when to start or stop the transmission of data from the PMU. The data format for this primarily consists of the time-tag and the command code, which informs the PMU of the action that needs to be taken.

\section{Communication Issues}

\section{A. Communication Options Available}

Communication links used by WAMS include both wired (telephone lines, fiber-optics, power lines) and wireless (satellites) options. Delays associated with the link act as a crucial indicator to the amount of time-lag that takes place before action is initiated. The delays are an important aspect and should be incorporated into any power system design or analysis, as excess delays could ruin any control procedures adopted to stabilize the power grid. The communication options for WAMS include the following:

Telephone lines: Telephone lines still form the backbone of utility communications. These offer data rates of up to about $56 \mathrm{kbps}$ (analog). In substations, due to the need for isolation circuits, this speed drops to around $9.6 \mathrm{kbps}$. The main advantage of using phone lines is that they are easy to set up and economical to use. Dial-up or leased lines could be considered, though the latter would be a slightly more expensive choice.
Fiber-optic cables: Many utilities are choosing fiberoptic cables for communication purposes and the demand for this medium is growing at a fast rate. These offer data rates ranging from 50 million to 1 billion bits per second. A small part of the bandwidth can be utilized for usage in WAMS. The remaining bandwidth can be used by the utilities for providing secondary services like Internet access and videoconferencing, which would provide for additional revenue generation [8]. The advantages of using fiber-optics include its immunity to RF \& atmospheric interference, and the massive bandwidth that it provides, which can be used by the utilities for other telecommunication needs. The disadvantage of using fiber-optics is its high initial investment and the lack of long haul digital fiber-optic systems expertise within many utilities [9].

Satellites: Low-earth orbiting (LEO) satellites can also be used to exchange data between the PMUs and the SPCs. The disadvantages of using a satellite include its high cost, narrow bandwidth, and associated link delays. Remote substation SCADA is one area where satellites have been used effectively. Among utilities, Southern California Edison (SCE) was the first to deploy an antennaterminal satellite system for substation SCADA [10].

Power lines: Power line communication (PLC) is a new technique that is fast emerging and can be considered for use in WAMS offering data rates in the range of 4 Mbps via the electricity supply grid. Power line technology uses the medium and low voltage electric supply grid for transmission of data and voice. It can be used for both WAMS communication requirements and substation networking [11]. Using spread spectrum techniques like direct sequence spread spectrum (DSSS) and frequency hopping spread spectrum (FHSS) or orthogonal frequency division multiplexing (OFDM) can result in tolerance of self interference due to multipath propagation and also interference due to co-channel or adjacent channel signals, thereby improving bit error rate (BER)[12].

Microwave links: Point to point microwave links are also being used by utilities to a great extent. Microwave links provide a better option as compared to leased lines, since they are easy to set up and are highly reliable. Digital microwave links are set to replace analog links in the near future. Digital microwave links fall into two categories: frequency bands less than $10 \mathrm{GHz}$ and frequency bands greater than $10 \mathrm{GHz}$. Bands which are below $10 \mathrm{GHz}$ have long propagation distances up to 30 miles. Bands over $10 \mathrm{GHz}$ are distance limited to less than five miles. The main disadvantages of using microwave links are signal fading and multipath propagation. To serve the needs of the utilities, high-speed data rate capability and noise immunity of digital microwave links can be prefered over analog microwave links [9]. Analog microwave links are fast becoming obsolete in today's digital era. 


\section{B. Communication Delay Causes}

Although more and more control systems are being implemented in a distributed fashion with networked communication, the unavoidable time delays in such systems impact the achievable performance [13]. Delays due to the use of PMUs and the communication link involved are due primarily to the following reasons:

Transducer delays: Voltage transducers (VT) and current transducers (CT) are used to measure the rms voltages and currents respectively, at the instant of sampling. Window size of the DFT: Window size of the DFT is the number of samples required to compute the phasors using DFT.

Processing time: The processing time required in converting the transducer data into phasor information with the help of DFT.

Data size of the PMU output: Data size of the PMU message is the size of the information bits contained in the data frame, header frame and the configuration frame.

Multiplexing and transitions: Transitions between the communication link and the data processing equipment leads to delays that are caused at the instances when data is retrieved or emitted by the communication link.

Communication link involved: The type of communication link and the physical distance involved in transmitting the PMU output to the central processing unit can add to the delay.

Data concentrators: Data concentrators are primarily data collecting centers located at the central processing unit and are responsible for collecting all the PMU data that is transmitted over the communication link.

\section{Delay Calculations}

Delay calculations form an important aspect of WAMS. These delays indicate the viability of a particular communication medium, since large communication delays amount to slower controller actions that can correct power grid instabilities and oscillations. Communication delay can be expressed as:

$$
\tau=\tau_{f}+\tau_{p}+\frac{L}{R}+\theta
$$

where $\tau$ is the total link delay, $\tau_{f}$ is the fixed delay associated with transducers used, DFT processing, data concentration and multiplexing, $\tau_{p}$ is the link propagation delay, $L$ is the amount of data transmitted, $R$ is the data rate of the link, and $\theta$ is the associated random delay jitter.

Delay calculations can be made on the assumptions that there are 10 - 12 phasor measurements, each 4 bytes in length, 10 input status channels, each 2 bytes in length, and the combined delay caused by processing, concentrators, multiplexing and transducers is estimated to be around $75 \mathrm{~ms}$. This is the fixed delay and is independent of the communication medium used.
TABLE I

DELAY CALCULATIONS ASSOCIATED WITH VARIOUS COMMUNICATION LINKS

\begin{tabular}{|l||c|}
\hline Communication link & $\begin{array}{c}\text { Associated delay- } \\
\text { one way (milliseconds) }\end{array}$ \\
\hline Fiber-optic cables & $\approx 100-150$ \\
\hline Digital microwave links & $\approx 100-150$ \\
\hline Power line (PLC) & $\approx 150-350$ \\
\hline Telephone lines & $\approx 200-300$ \\
\hline Satellite link & $\approx 500-700$ \\
\hline
\end{tabular}

The propagation delay is dependent on the medium and thus is a function of both the medium and the physical distance separating the individual components of WAMS. We have assumed that media like fiber-optic cables, power lines, and telephone lines, on an average, have a propagation delay of around $25 \mathrm{~ms}$. In the case of LEO satellites, the propagation delay could be as high as $200 \mathrm{~ms}$, because of the distance involved and gateways that connect terrestrial networks to the satellite. There would be further delay involved if there is some processing done at the gateways or by the satellite itself. There is also additional delay caused by the amount of data that has to be transported across the medium and the data rate of the medium.

The analog to digital converter (ADC) in the PMU has 16-bit resolution and sampling is assumed at 720 samples per second. For the PMU, it is estimated that the length of the data frame is around 640 bits, the length of the header frame is around 200 bits and the length of the configuration frame is around 2800 bits. The normal operation of the PMU requires only the transmission of the data file. The header and configuration files can be transmitted at equipment startup or on a request basis. For worst case delay scenarios, we have assumed that there is a regular transmission of the header and configuration files along with the data files.

Considering the total length of the PMU packet, the delay caused due to the amount of data transmitted and the data rate, can then be estimated to be around 125 ms, considering a telephone line channel with an average capacity of $33.6 \mathrm{kbps}$. In the case of substations, where isolation circuits are required, the telephone line channel capacity could drop to as low as $9.6 \mathrm{kbps}$, creating a bottleneck, and thereby increasing the delay to more than $200 \mathrm{~ms}$. Even though power lines can reach speeds of up to $4 \mathrm{Mbps}$, delay calculations due to the amount of data transmitted and the data rate, can be assumed to be similar to telephone line delay calculations. This assumption is valid because power lines suffer from an enormous amount of interference and satisfactory BER performance would require data rates in the range of a few kbps. Fiber- 
optic cables do not contribute to the delay associated with the length of the PMU packet because of their high data rates, and hence the associated delay can be considered to be negligible. The delay analysis of digital microwave links would be similar to that of fiber-optic channels because the data rate for the former can be considered to be unlimited for all practical purposes [9]. The length of the PMU packet also introduces additional delay in the case of satellites because of narrow bandwidth channels, noise, and interference.

Table I indicates the delays of various communication links when using PMUs in a WAMS environment and could provide useful delay statistics that can be integrated into simulation and performance analysis of WAMS. All the above mentioned communication link options can provide for almost real-time correction of unstable situations that could lead to large area cascaded outages. The advantages and disadvantages of each communication link have to be weighed by the utilities and then the right link has to be chosen based on the required control dynamics and operating economics of the power system.

\section{CONCLUSIONS}

Dynamic monitoring and control of power system networks using the concept of WAMS increases transmission efficiency and reliability. WAMS is an appropriate system for business support in an unbundled and open-access utility environment and is designed for an open-access market where production and transmission patterns will more often change than in a closed market. WAMS also helps create maximum profitability by reducing downtime and by optimizing system performance.

PMUs in a WAMS environment, provide for almost real-time monitoring and control of grid stability considering the fact that action can be initiated in a matter of milliseconds. Future work should involve integrating the delays associated with PMUs, for different communication links, along with other communication applications, into simulations that analyze the technical performance and economic viability of the various communication links that can be used in WAMS.

\section{ACKnowledgment}

This work was sponsored in part by a US DOE/EPSCoR WV State Implementation Award.

\section{REFERENCES}

[1] C. Rehtanz and J. Bertsch, "A new wide area protection system," in 2001 IEEE Porto Power Tech Conference, vol. 4 (Porto, Portugal), pp. 186-191, September 2001.

[2] A. G. Phadke, "Synchronized phasor measurements in power systems," IEEE Computer Applications in Power, vol. 6 , pp. 10-15, April 1993.

[3] A. G. Phadke, J. S. Thorp, and K. J. Karimi, "State estimation with phasor measurements," IEEE Transactions on Power Systems, vol. 1, 1986.

[4] D. G. Hart, D. Novosel, M. Subramanian, and M. Ingram, "Real-time wide area measurements for adaptive protection and control," in NSF/DOE/EPRI Workshop on Future Research Directions for Complex Interactive Electric Networks, (Washington, D.C.), November 2000.

[5] V. Centeno, A. Phadke, A. Edris, J. Benton, M. Gaudi, and G. Michael, "An adaptive out-of-step relay," IEEE Transactions on Power Delivery, vol. 12, pp. 61-67, January 1997.

[6] A. G. Phadke, "Computer relaying - its impact on improved control and operation in power systems," IEEE Computer Applications in Power, vol. 1, pp. 5-10, October 1988.

[7] IEEE Standards Board, IEEE Standard for Synchrophasors for Power Systems, December 1995. IEEE Std. 1344-1995.

[8] L. Lamarre, "At home with communications," EPRI Journal, pp. 7-15, January/February 1997.

[9] Radio Subcommittee of IEEE Power System Communications Committee, "Analog/digital microwave considerations for electric/gas utilities," IEEE Transactions on Power Delivery, vol. 8, pp. 798-815, July 1993.

[10] T. Moore, "Utility communications go into orbit," EPRI Journal, 1999

[11] D. Radford, "Spread spectrum data leap through ac wiring," IEEE Spectrum, pp. 48-53, November 1996.

[12] K. M. Dostert, "Frequency hopping spread spectrum modulation for digital communications over power lines," IEEE Journal in selected areas in Communications, vol. 8, pp. 700-710, May 1990.

[13] J. K. Yook, D. M. Tilbury, and N. R. Soparkar, "Design methodology for distributed control systems to optimize performance in the presence of time delays," in Proc. of the American Control Conference, (Chicago, Illinois), pp. 1959-1964, June 2000. 